\title{
Ultrafast Exciton Population, Relaxation, and Decay Dynamics in Thin Oligothiophene Films
}

\author{
E. Varene, L. Bogner, C. Bronner, and P. Tegeder* \\ Fachbereich Physik, Freie Universität Berlin, Arnimallee 14, 14195 Berlin, Germany
}

(Received 24 February 2012; published 13 November 2012)

\begin{abstract}
Femtosecond time-resolved two-photon photoemission spectroscopy is utilized to determine the electronically excited states dynamics at the $\alpha$-sexithiophene (6T)/ $\mathrm{Au}(111)$ interface and within the $6 \mathrm{~T}$ film. We found that a photoinduced transition between the highest occupied molecular orbital and lowest unoccupied molecular orbital is essential in order to observe exciton population, which occurs within $100 \mathrm{fs}$. In thin $6 \mathrm{~T}$ films, the exciton exhibits a lifetime of $650 \mathrm{fs}$. On a time scale of $400 \mathrm{fs}$, an energetic stabilization is observed leading to the formation of a polaron or electron trapping at defect states. The lifetime of this state is 6.3 ps. Coverage-dependent measurements show that apart from the excited state decay within the film, a substrate-mediated relaxation channel is operative. The present study demonstrates that two-photon photoemission spectroscopy is a powerful tool to investigate the whole life cycle from creation to decay of excitons in an organic semiconductor.
\end{abstract}

DOI: 10.1103/PhysRevLett.109.207601

PACS numbers: 79.60.Fr, 71.35.- $-\mathrm{y}, 78.47 . j \mathrm{~d}$

Organic semiconductors are being explored for a number of fascinating applications in optoelectronic devices such as organic photovoltaic cells, organic light emitting diodes, or organic thin film transistors [1,2]. A complete knowledge of the electronic structure and the dynamics of electronically excited states in potential molecule-based devices is necessary for improvement and optimization of the device performance. Thereby the exciton formation and decay dynamics at interfaces and within the molecular films are of particular relevance. Recently it was discussed by Koch et al. [3] that the choice of the appropriate experimental method to study exciton populations is important. They have emphasized that the existence of exciton signatures in absorption or luminescence spectra are due to a coherent excitonic polarization and are not a fingerprint of a real incoherent exciton population. For excitations above the band gap (interband absorption), fast Coulomb and phonon scattering results in polarization decay and the generation of electron-hole pair population on a femtosecond (fs) time scale [3]. Therefore fs timeresolved two-photon photoemission (2PPE) is a very powerful tool to study these processes. The first pump pulse excites a transition between the highest occupied molecular state and lowest unoccupied molecular state ("interband" adsorption), while the second pulse probes the electronically excited state. By varying the time delay between the pump and probe pulses from a few fs up to several picoseconds, it is thus possible to resolve the exciton population and decay dynamics.

In the present study we utilized fs time-resolved 2PPE to determine the charge carrier and exciton dynamics at the $\alpha$-sexithiophene (6T)/ $\mathrm{Au}(111)$ interface and within the 6T film. Oligothiophenes, $\pi$-conjugated chainlike planar molecules, are highly relevant for small-molecule-based solar cells, which are fabricated via vacuum deposition. In bulk heterojunction photovoltaic cells they possess very high efficiencies [4]. Several surface science studies focusing on the adsorption behavior of $6 \mathrm{~T}$ on noble metal surfaces exist, e.g. on $\mathrm{Au}(111)$ [5] and $\mathrm{Cu}(110)$ [6]. In addition, the occupied band structures of $6 \mathrm{~T}$ adsorbed on various noble metals have been determined [7-9]. Using 2PPE the electronic structure, i.e., both occupied and unoccupied molecular electronic as well as excitonic states, of 6T adsorbed on $\mathrm{Au}(111)$ have been observed. Therein the energetic position of the transport level and the exciton binding energy have been determined [10]. In contrast to time-resolved measurements ranging from femtoseconds to milliseconds to elucidate the decay dynamics of excitons in 6T films (thickness about $50-350 \mathrm{~nm}$ ) [11-13], investigations on the exciton formation, relaxation, and decay at $6 \mathrm{~T} / \mathrm{Au}(111)$ interfaces and within thin films are not known so far. A recent 2PPE study investigated the charge transport properties of $6 \mathrm{~T}$ and the dihexyl-substituted 6T (DH6T) adsorbed on $\operatorname{Ag}(111)$, respectively, and found a charge localization in DH6T within 230 fs while in 6T no localization was observed. The charge localization has been attributed to the hydrocarbon side chains [14]. The exciton decay dynamics at organic molecule-metal interfaces has been observed so far only for $\mathrm{C}_{60}$ films epitaxially grown on $\mathrm{Au}(111)$ and $\mathrm{Cu}(111)$ using 2PPE [15]. Additionally 2PPE has been used to probe charge transfer excitons on a surface of organic semiconductors, namely, pentacene and tetracene, respectively $[16,17]$.

In this Letter, we investigate the exciton formation and decay in $6 \mathrm{~T}$ on $\mathrm{Au}(111)$ as a function of 6T coverage using 2PPE. We found that an optically induced transition between the highest occupied molecular orbital (HOMO) and lowest unoccupied molecular orbital (LUMO) is the basic prerequisite in order to observe the population of a Frenkel exciton. The exciton generation occurs within 100 fs followed by an energetic stabilization on a time 
scale of around $400 \mathrm{fs}$ leading to a polaronic or trap state. Both the excitonic and polaron or trap state exhibit a coverage dependent lifetime, which in each case is associated with two decay channels, an intrinsic and a distancedependent (external) decay.

In our experiment, the $\mathrm{Au}(111)$ crystal was mounted on a liquid nitrogen cooled cryostat that, in conjunction with resistive heating, enables temperature control from 90 to $800 \mathrm{~K}$. The crystal was cleaned by standard procedure of cycles of $\mathrm{Ar}^{+}$sputtering and annealing. The 6T was dosed by means of a home-built effusion cell held at $500 \mathrm{~K}$ at a crystal temperature of $290 \mathrm{~K}$. The $6 \mathrm{~T}$ coverage was quantified by temperature-programmed desorption and work function measurements. Tunable fs laser pulses for the time-resolved 2PPE-measurements are supplied from a Ti:Sapphire oscillator in combination with a $300 \mathrm{kHz}$ regenerative amplifier and optical parametrical amplifiers [18].

Using photon energy-dependent 2PPE, we recently determined the electronic structure of $6 \mathrm{~T}$ adsorbed on $\mathrm{Au}(111)$ [10], which is summarized in Fig. 1. The exciton state is found $0.9 \mathrm{eV}$ below the LUMO. Although the exciton is not a single particle state but a quasiparticle formed by a correlated electron and hole bound through Coulomb interaction, in 2PPE the final state being an electron and an ionized molecule, it is often simpler to assign the binding energy to the electron. It is essential to point out that only excitations with photon energies enabling a HOMO-LUMO transition (resonant excitation), i.e., around $2.9 \mathrm{eV}$, permitted the observation of the exciton generation in 2PPE. This is in accordance with a real

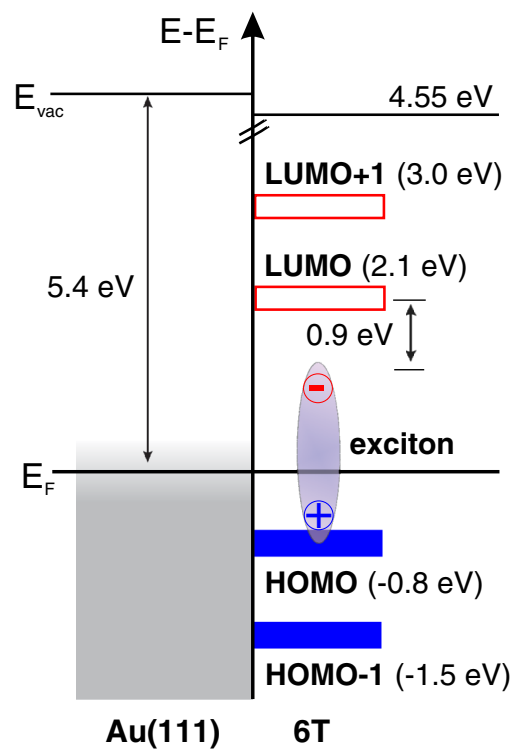

FIG. 1 (color online). Energetic position and assignment of 6T-derived photoemission spectral features at a coverage of 1 monolayer (ML) 6T adsorbed on $\mathrm{Au}(111)$. The Fermi level of $\mathrm{Au}(111)$ serves as reference. The exciton binding energy is $0.9 \mathrm{eV}$. exciton population process [3]. Chan et al. [19] have applied 2PPE to study the excited state dynamics in pentacene or fullerene bilayers after optical excitation and observed multiple exciton generation. However, we excite the system with $3 \mathrm{eV}$ photons and observe the feature attributed to a Frenkel exciton at a final state energy of $2 \mathrm{eV}$ above the HOMO; thus, we can rule out the formation of two or more electron-hole pairs from the absorption of one photon. Moreover, our assignment is in very good agreement with the results of Frolov et al. [20] who have found that Frenkel excitons in $6 \mathrm{~T}$ are characterized by a stimulated emission band at $2 \mathrm{eV}$.

In the following we focus on the excited state dynamics, in particular, the population and decay dynamics of the Frenkel exciton. In the low-coverage regime (1-2 ML), the lifetimes for electrons in the molecular excited states, viz. the LUMO and LUMO +1 , possess values that are below the limit of our experimental resolution $(\tau<10 \mathrm{fs})$. These ultrashort lifetimes point towards a strong electronic coupling between 6T and $\mathrm{Au}(111)$, allowing for efficient back transfer of electrons to the metal substrate. At metallic surfaces such short lifetimes of excited states, i.e., in the range of fs, have been found for several adsorbates $[15,18,21-23]$. In contrast, the exciton exhibits a

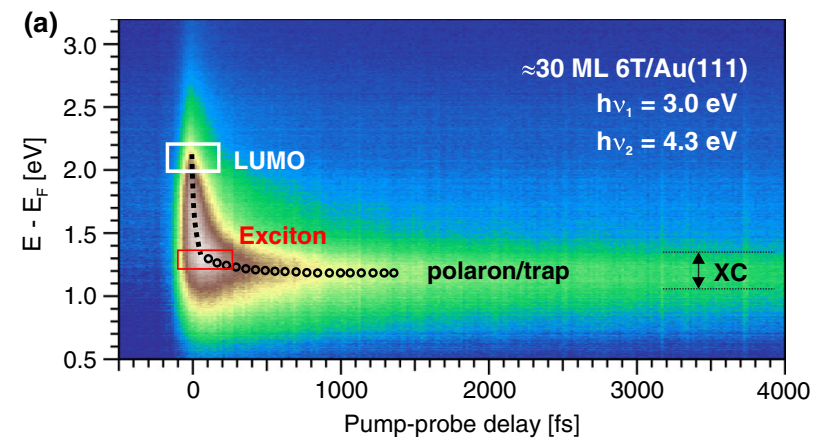

(b)
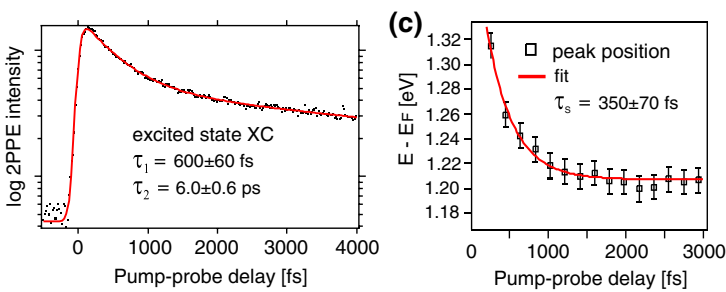

FIG. 2 (color online). Two-dimensional spectrum of timeresolved 2PPE measurements recorded with $h \nu_{1}=3.0 \mathrm{eV}$ and $h \nu_{2}=4.3 \mathrm{eV}$. The dashed line in (a) indicates the energetic stabilization of electrons leading to the formation of the exciton, and the dotted line represents the energetic stabilization of electrons caused by the formation of polarons or at defect sites trapped electrons (see text). (b) Cross correlation traces of the 2PPE intensity integrated over the excited state peak intensities [XC energy range in (a)] with a biexponential fit that gives the indicated lifetimes. (c) Maximum peak intensity of the exciton state as a function of pump-probe delay that yields the indicated energy stabilization time due to polaron formation or localization at defect sites. 
pronounced lifetime as can clearly be seen in Fig. 2(a) for $30 \mathrm{ML} 6 \mathrm{~T} / \mathrm{Au}(111)$. In this false color two-dimensional representation, the 2PPE intensity at particular energy with respect to the Fermi level, $E_{F}$, as a function of time delay $(\Delta t)$ between the two laser pulses $h \nu_{1}=3.0 \mathrm{eV}$ and $h \nu_{2}=$ $4.3 \mathrm{eV}$ is displayed. The cross correlation curve for the energy region of the excited state [XC in Fig. 2(a)] is shown in Fig. 2(b). The solid line corresponds to a fit used to determine lifetimes. The fit model contains a sech ${ }^{2}$ function representing the laser pulse duration convoluted with the response function of the intermediate state. The response function is biexponential yielding the indicated lifetime values $\tau_{1}$ and $\tau_{2}$, which correspond to two different states. A detailed discussion about the origin of these states will be given below.

Returning to the time-resolved 2PPE spectra shown in Fig. 2(a), a fast energetic stabilization of electrons (dashed line) leading to the formation of the Frenkel exciton is clearly seen. In addition, the excitonic state stabilizes on a slower time scale (dotted line). The first fast process, the stabilization of around $0.9 \mathrm{eV}$, occurs within $100 \pm 20 \mathrm{fs}$ [see Fig. 2(a)]. We interpret this observation as follows: Optical excitation with $3.0 \mathrm{eV}$ photons leads to an intramolecular HOMO-LUMO transition, followed by the decay of the polarization and formation of the exciton population (correlated electron-hole pair) that occurs on a femtosecond time scale [3]. The transition of the electron from a bonding (the HOMO) to an antibonding state (the LUMO) is a very efficient source for the stimulation of molecular vibrations; therefore, the exciton binding energy of $0.9 \mathrm{eV}$ dissipates to molecular vibrations on a time scale of the inverse of a vibrational quantum $(\leq 100 \mathrm{fs}$, for instance the C-S-C ring deformation mode at $700 \mathrm{~cm}^{-1}$ [24], which corresponds to a period of $48 \mathrm{fs}$ ). On the other hand, coupling of the exciton to molecular vibrations (electron-nuclear interaction) can lead to the generation of a polaron. This process may be responsible for the observed energetic stabilization of around $0.1 \mathrm{eV}$, which proceeds on a time scale of $350 \pm 70$ fs [see Fig. 2(c)]. In addition to the decrease in electron energy, this kind of localization due to the polarization in the nuclear coordinates should be accompanied by a time-dependent disappearance of parallel dispersion [14,25]. However, the photoemission of the electron that is intrinsic to $2 \mathrm{PPE}$ breaks the exciton, and thus we cannot measure the dispersion of this excitonic state. Besides polaron formation being responsible for the energetic stabilization, the electron may be trapped into a defect site [26]. Localization by defects has been shown to occur on a hundred fs time scale in particular systems [27]. Distinguishing between electrons trapped into a defect site and a self-localizing polaron is difficult because of the similar time scales for both formation processes.

In order to gain further insights into the exciton decay, we investigated the dynamics as a function of $6 \mathrm{~T}$ coverage. The cross correlation curves for the energy region of the excited state (exciton and polaron or trap) as a function of coverage are presented in Fig. 3. Both lifetime values $\tau_{1}$ and $\tau_{2}$ exhibit a clear dependence on layer thickness: $\tau_{1}$ increases from $40 \pm 10$ fs at $3 \mathrm{ML}$ to $650 \pm 65 \mathrm{fs}$ at $30 \mathrm{ML}, \tau_{2}$ rises from $200 \pm 20 \mathrm{fs}(3 \mathrm{ML})$ to $6 \pm 0.6 \mathrm{ps}(30 \mathrm{ML})$. The results of the fits as a function of film thickness are summarized in Figs. 3(b) and 3(c). The solid lines are fits using a simple exponential function. The asymptotic values are $650 \pm 50$ fs for $\tau_{1}$ and $6.3 \pm 0.6 \mathrm{ps}$ for $\tau_{2}$, respectively. As it has been discussed for the monoexponential exciton decay in $\mathrm{C}_{60}$ adsorbed on $\mathrm{Au}(111)$ and $\mathrm{Cu}(111)$ [15], respectively, a coverage-dependent decay time indicates that the exciton decays via two relaxation channels, an intrinsic and a distance-dependent channel. The intrinsic decay rate corresponds to the decay in the bulk material and the distance- dependent (external) rate to quenching by the metal substrate. Accordingly for $6 \mathrm{~T} / \mathrm{Au}(111)$, the coverage dependence of both decay times indicate that each of them contains at least two relaxation channels, an intrinsic and an external channel. The intrinsic decays for bulk $6 \mathrm{~T}$ are
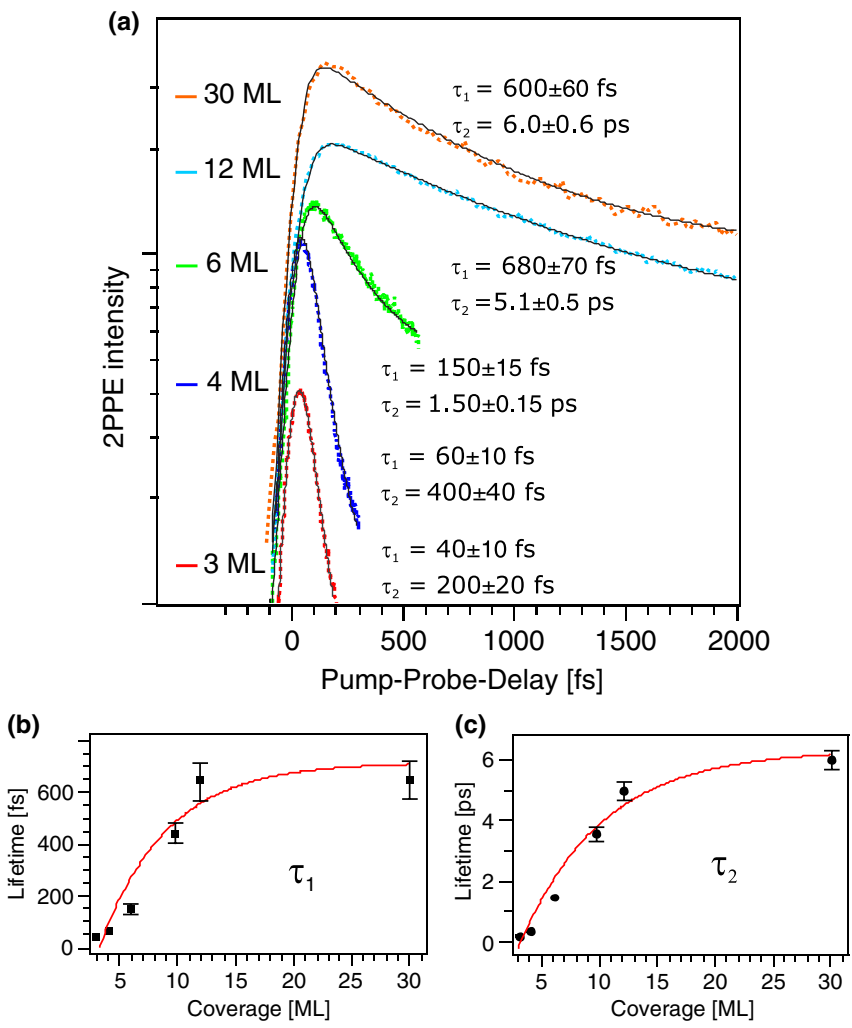

FIG. 3 (color online). (a) Cross correlation traces of the 2PPE intensity integrated over the excited state peak intensities (exciton and polaron or trap) as a function of $6 \mathrm{~T}$ coverage on a logarithmic intensity axis. The lines represent fits for the biexponential decay, which yields the indicated lifetimes. The dependence on film thickness of the transient lifetime (b) $\tau_{1}$ and (c) $\tau_{2}$. 


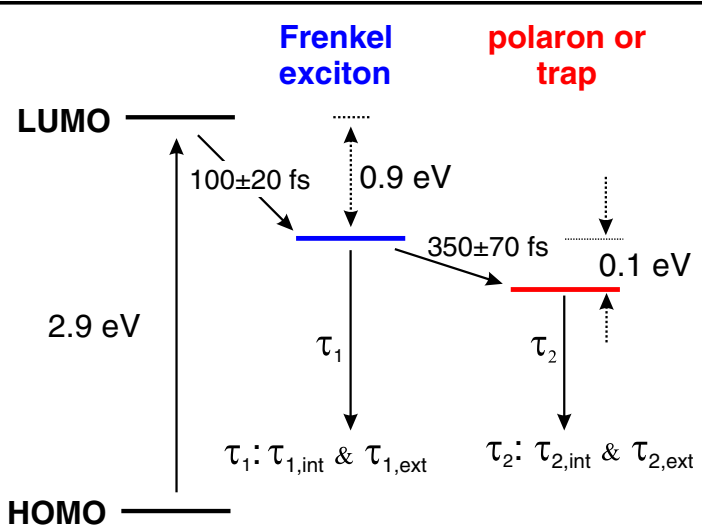

FIG. 4 (color online). Formation and decay processes of a Frenkel exciton in sexithiophene adsorbed on $\mathrm{Au}(111) . \tau_{\text {int }}$ indicates the intrinsic (for bulk 6T) and $\tau_{\text {ext }}$ the external (distance-dependent) lifetime (see text).

related to the asymptotic values of $650 \pm 50 \mathrm{fs}$ for $\tau_{1}$ and $6.3 \pm 0.6$ ps for $\tau_{2}$ [Figs. 3(b) and 3(c)].

Figure 4 summarizes the exciton formation and decay processes as determined in our time-resolved photoemission study. We attribute the two lifetime values $\tau_{1}$ and $\tau_{2}$ obtained from the biexponential decay (see Fig. 2) to originate from the decay of the singlet Frenkel exciton $\left(\tau_{1}\right)$ and the polaron or defect state $\left(\tau_{2}\right)$, respectively. As discussed above, both values contain an intrinsic $\left(\tau_{\text {int }}\right.$, for bulk $6 \mathrm{~T}$ ) and external ( $\tau_{\text {ext }}$, coverage-dependent decay to metal states) decay. Before addressing the coveragedependent lifetime, we discuss the intrinsic lifetime of the Frenkel exciton and the polaron or defect state in bulk $6 \mathrm{~T}$ by comparing with time-resolved studies known from literature. Most studies [11-13] determined the dynamical processes after optical excitation of higher lying electronic states compared to the formation and decay of the lowest (first) Frenkel exciton state investigated here. In addition, almost all of these experimental results revealed dynamic processes on much longer time scales. For the singlet Frenkel exciton, we observe a lifetime of $\tau_{1, \text { int }}=$ $650 \pm 50$ fs. In comparison, a value of $\approx 1$ ps has been found for the decay in $6 \mathrm{~T}$ films using picosecond fluorescence spectroscopy [12]. The lifetime of the polaron or defect state is $\tau_{2 \text {,int }}=6.3 \pm 0.6 \mathrm{ps}$, thus one order of magnitude longer than the first exciton state. The coverage- or distance-dependent lifetime $\left(\tau_{\text {ext }}\right)$ is associated with backtransfer of electrons to the metallic substrate (substratemediated quenching). With increasing 6T coverage, the probability density of the excited state (exciton, polaron, or trap) is further away from the metal-molecule interface. As a result, its coupling (wave function mixing) to the $\mathrm{Au}$ surface decreases and the electron transfer rate decreases [15].

In conclusion, using fs time-resolved 2PPE we were able to elucidate the exciton population and decay dynamics in sexithiophene adsorbed on $\mathrm{Au}(111)$. The formation of the exciton takes place on a femtosecond time scale following optical excitations higher than the HOMO-LUMO gap energy. In bulk 6T, the exciton either recombines, it possesses a lifetime of $650 \mathrm{fs}$, or energetic stabilization results in generation of a polaron or trapped charge (with a lifetime of $6.3 \mathrm{ps}$ ). Both the exciton and the polaron or trap state lifetimes are coverage dependent indicating that an intrinsic (for bulk 6T) and an external relaxation channel exist. Our study shows that 2PPE is a powerful tool to determine exciton population and decay dynamics. Therefore, this method promises to provide a link between optical and electron spectroscopy. The creation and decay of excitons are important elementary processes in organic semiconductors. Precise insight into these processes is certainly essential for optimization of organic-moleculebased optoelectronic devices.

Funding by the Deutsche Forschungsgemeinschaft (DFG) through the priority program SPP 1355 and collaborative research center SFB 658 is gratefully acknowledged.

*Corresponding author. petra.tegeder@physik.fu-berlin.de

[1] H. E. Katz and J. Huang, Annu. Rev. Mater. Res. 39, 71 (2009).

[2] K. Walzer, B. Maennig, M. Pfeiffer, and K. Leo, Chem. Rev. 107, 1233 (2007).

[3] S. W. Koch, M. Kira, G. Khitrova, and H. M. Gibbs, Nature Mater. 5, 523 (2006).

[4] R. Fitzner, E. Reinhold, A. Mishra et al., Adv. Funct. Mater. 21, 897 (2011).

[5] M. Kiel, K. Duncker, C. Hagendorf, and W. Widdra, Phys. Rev. B 75, 195439 (2007).

[6] M. Kiguchi, S. Entani, K. Saiki, and G. Yoshikawa, Appl. Phys. Lett. 84, 3444 (2004).

[7] I. G. Hill, A. Kahn, Z. G. Soos, and R. A. Pascal Jr., Chem. Phys. Lett. 327, 181 (2000).

[8] N. Koch, G. Heimel, J. Wu, E. Zojer, R. L. Johnson, J.-L. Brédas, K. Müllen, and J. P. Rabe, Chem. Phys. Lett. 413, 390 (2005).

[9] M. Grobosch and M. Knupfer, Adv. Mater. 19, 754 (2007).

[10] E. Varene, I. Martin, and P. Tegeder, J. Phys. Chem. Lett. 2, 252 (2011).

[11] G. Lanzani, S. V. Frolov, P. A. Lane, Z. V. Vardeny, M. Nisoli, and S. De Silvestri, Phys. Rev. Lett. 79, 3066 (1997).

[12] K. Watanabe, T. Asahi, H. Fukumura, H. Masuhara, K. Hamano, and T. Kurata, J. Phys. Chem. B 101, 1510 (1997).

[13] G. Urbasch, H. Giessen, M. Murgia, R. Zamboni, and R. F. Mahrt, J. Phys. Chem. B 104, 6536 (2000).

[14] J. E. Johns, E. A. Muller, J. M. J. Frechet, and C. B. Harris, J. Am. Chem. Soc. 132, 15720 (2010).

[15] G. Dutton, D. P. Quinn, C. D. Lindstrom, and X.-Y. Zhu, Phys. Rev. B 72, 045441 (2005). 
[16] M. Muntwiler, Q. Yang, W. A. Tisdale, and X.-Y. Zhu, Phys. Rev. Lett. 101, 196403 (2008).

[17] Q. Yang, M. Muntwiler, and X.-Y. Zhu, Phys. Rev. B 80, 115214 (2009).

[18] S. Hagen, Y. Luo, R. Haag, M. Wolf, and P. Tegeder, New J. Phys. 12, 125022 (2010).

[19] W. Chan, M. Ligges, A. Jailaubekov, L. Kaale, L. MiajaAvila, and X.-Y. Zhu, Science 334, 1541 (2011).

[20] S. V. Frolov, Ch. Kloc, B. Batlogg, M. Wohlgennant, X. Jiang, and Z. V. Vardeny, Phys. Rev. B 63, 205203 (2001).

[21] A. Yang, S. T. Shipman, S. Garrett-Roe, J. Johns, M. Strader, P. Szymanski, E. Muller, and C. B. Harris, J. Phys. Chem. C 112, 2506 (2008).
[22] M. Wolf and P Tegeder, Surf. Sci. 603, 1506 (2009).

[23] C. Bronner, M. Schulze, S. Hagen, and P. Tegeder, New J. Phys. 14, 043023 (2012).

[24] G. Louran, J. P. Buisson, S. Lefrant, and D. Fichou, J. Phys. Chem. 99, 11399 (1995).

[25] A. D. Miller, I. Bezel, K. J. Gaffney, S. Garrett-Roe, S. H. Liu, P. Szymanski, and C. B. Harris, Science 297, 1163 (2002).

[26] L. G. Kaake, P. F. Barbara, and X.-Y. Zhu, J. Phys. Chem. Lett. 1, 628 (2010).

[27] U. Bovensiepen, C. Gahl, J. Stähler, M. Bockstedte, M. Meyer, F. Baletto, S. Scandolo, X.-Y. Zhu, A. Rubio, and M. Wolf, J. Phys. Chem. C 113, 979 (2009). 